\title{
Ultracold neutrons extracted from a superfluid-helium converter coated with fluorinated grease
}

\author{
O. Zimmer ${ }^{1,2, a}$, P. Schmidt-Wellenburg ${ }^{1,2,4,5}$, M. Fertt ${ }^{1,4,5}$, H.-F. Wirth ${ }^{1,6}$, M. Assmann ${ }^{3}$, J. Klenke ${ }^{3}$, \\ B. van den Brandt ${ }^{4}$ \\ ${ }^{1}$ Physik-Department E18, Technische Universität München, 85748 Garching, Germany \\ ${ }^{2}$ Institut Laue-Langevin, B.P. 156, 38042 Grenoble, France \\ ${ }^{3}$ Forschungsreaktor München FRM II, Lichtenbergstrasse 1, 85747 Garching, Germany \\ ${ }^{4}$ Paul Scherrer Institut, 5232 Villigen PSI, Switzerland \\ ${ }^{5}$ Present address: Paul Scherrer Institut, 5232 Villigen PSI, Switzerland \\ ${ }^{6}$ Present address: Ludwig Maximilians Universität München, Am Coulombwall 1, 85748 Garching, Germany
}

Received: 17 October 2009 / Revised: 25 February 2010 / Published online: 27 April 2010

(C) The Author(s) 2010. This article is published with open access at Springerlink.com

\begin{abstract}
We report experiments on the production of ultracold neutrons (UCN) in a converter of superfluid helium coated with fluorinated grease (fomblin). We employed our special technique of window-free extraction of accumulated UCN from the superfluid helium, in which they were produced by downscattering neutrons of a cold beam from the Munich research reactor. The fomblin-coating reduced the time constant for UCN passage through the extraction hole by a factor three compared to our previous experiment employing an uncoated stainless steel vessel. A time-of-flight measurement of the cold neutron spectrum incident on the converter, combined with a gold foil activation, allowed us to determine both the single-phonon and multi-phonon contributions to the UCN production. The UCN production rate is in reasonable agreement with the theoretical expectation.
\end{abstract}

\section{Introduction}

Ultra-cold neutrons (UCN) are defined by their property to undergo total reflection under any angle of incidence when impinging on a wall with a positive material optical potential. The main origin of this potential is coherent neutron scattering by atomic nuclei in bulk matter. For well-selected wall materials it enables trapping of neutrons with energy up to about $300 \mathrm{neV}$. In bottles with small cross sections for neutron absorption and inelastic scattering and a typical mean free path of a few tens of centimetres, UCN can be stored and manipulated for times as long as several hundred seconds. Owing to this feature UCN have become a very

a e-mail: zimmer@ill.fr useful tool and object in various fundamental investigations, some with important implications for particle physics and cosmology (see the books [1,2] for an introduction to the physics of UCN).

Longstanding and more actively pursued than ever is the search for the neutron electric dipole moment with the goal to find CP-violation beyond the standard model of particle physics [3, 4]. A second neutron property being intensely addressed in several projects is the neutron lifetime. A value more accurate than currently available is required for a detailed understanding of big bang nucleosynthesis $[5,6]$, and to investigate strength and structure of the semi-leptonic weak interaction within the first quark family $[7,8]$. UCN have also proven to be useful to demonstrate quantum mechanical properties of the neutron such as bound states above a horizontal flat mirror in the earth's gravitational field [9]. Recent, new topics are searches for neutron-mirror neutron transitions [10, 11], and for axion-like particles [12-14]. The most intense source for user experiments with UCN, at the Institut Laue Langevin in Grenoble [15], provides UCN densities not exceeding a few 10 per $\mathrm{cm}^{3}$. In order to improve counting statistical accuracy of experiments, new UCN sources are being developed in many laboratories around the world [16-22].

An elegant method to produce UCN employs a converter of superfluid ${ }^{4} \mathrm{He}$ in a beam of cold neutrons [23]. The kinematics defined by the dispersion relations of helium and the free neutron enables down-scattering of cold neutrons with an energy around $1.0 \mathrm{meV}$ (corresponding to a neutron wavelength of $\lambda^{*}=0.89 \mathrm{~nm}$ ) to ultracold energies via emission of a single phonon. In addition, multi-phonon processes occur which contribute to the integral $\mathrm{UCN}$ production rate for a wide range of incident neutron energies [24]. At low 
temperatures the probability for up-scattering is strongly suppressed by the Boltzmann factor according to detailed balance. Therefore, and since pure ${ }^{4} \mathrm{He}$ has no cross section for neutron absorption, a large density of UCN may build up in a converter with reflective walls. The storage lifetime $\tau$ can of course not exceed a limit close to $900 \mathrm{~s}$ set by the neutron beta decay lifetime.

In a recent experiment with a new prototype apparatus we have demonstrated that UCN can be efficiently extracted from a superfluid ${ }^{4} \mathrm{He}$ converter into a neutron guide at room temperature, after having accumulated them to saturation density [25]. This became possible by vertically extracting the UCN through a cold mechanical valve situated above the helium bath. In contrast to a previous attempt to extract accumulated UCN horizontally [26], no loss-prone gaps or windows are required in our method, which now opens the door to apply UCN production in superfluid ${ }^{4} \mathrm{He}$ for a wide range of experiments and possibly as a user facility. As a first step in this direction an upgrade of the prototype will be installed at an intense monochromatic neutron beam at the ILL in order to feed UCN to an experiment to continue the study of bound quantum states above a flat mirror [27]. The design of our cryostat takes into account that no extraordinary cooling power is required at such a location of a UCN source and thus can be kept transportable and easy to operate. With a short cooling cycle of a few days, our system is indeed much more flexible than an earlier apparatus [28], which however was designed to be installed close to the target of a spallation source where significant heat load occurs due to radiation.

To gain first experience with our apparatus and keep things simple for the beginning, the extraction guide and a large part of the UCN converter vessel were made of stainless steel. Some more details of the design of the vessel and the extraction valve will be given below. Stainless steel is not the best choice with respect to maximum neutron optical potential and small absorption cross section, and will be replaced by ceramics from $\mathrm{BeO}$ in the upgrade [27]. Although not yet providing optimum performance as a UCN source, first runs with the present system allowed us to estimate the UCN production rate and study temperature-dependent storage properties of the converter.

In continuation of our investigations performed at the neutron beam "NL1" at the position "MEPHISTO" of the research reactor FRM II in Munich, we present new results obtained with the converter vessel coated with fluorinated grease (fomblin) which reduces the UCN absorption. Despite the smaller neutron optical potential of the coating (115 neV versus $184 \mathrm{neV}$ for stainless steel), and to our very surprise, we observed much faster extraction time constants, accompanied with higher UCN count rates. The present experiments were complemented by a time-of-flight (TOF) measurement of the incident cold neutron beam to enable a better comparison with the theoretical UCN production rate. As in prior experiments performed by other groups [20, 21, $29,30]$, we found the UCN production rate to agree reasonably well with the theoretical expectation.

\section{Apparatus}

A short description of the whole apparatus can be found in ref. [25] where a schematics of the converter vessel and the UCN extraction system is shown. Here we give more details. Figure 1 shows a design drawing of the UCN vessel with part of the extraction guide. It is comprised of a " $T$ " section (item (1) in Fig. 1), into which extension tubes with wall thickness $0.5 \mathrm{~mm}$ were plugged on both sides (the shorter plug (2) is visible in the detail magnifications). These parts consist of electropolished stainless steel with neutron optical potential $V_{\mathrm{SS}}=(184 \pm 4) \mathrm{neV}$, and with an assembled total length of $696 \mathrm{~mm}$ and inner diameter $66 \mathrm{~mm}$. Two $0.125 \mathrm{~mm}$ thick windows (3) made of $\mathrm{Ni}\left(V_{\mathrm{Ni}}=252 \mathrm{neV}\right)$ close the vessel on both ends, and a flange including the flap valve ((4), a photograph is shown in Fig. 2) closes the third port of the "T" piece.

Tightness for superfluid ${ }^{4} \mathrm{He}$ is provided by an outer jacket comprised of two aluminium pipes with inner diameter $70 \mathrm{~mm}$, each closed by welded $0.5 \mathrm{~mm}$ thick $\mathrm{Al}$ windows (5) on the outer end, and indium-sealed onto flanges of the " $T$ " piece on the other end (see left detail magnification in Fig. 1). The Ni windows are pressed by springs onto the outer ends of the stainless steel tubes. Although the UCN vessel itself is not helium-tight, four holes with diameter $0.5 \mathrm{~mm}$ were drilled in the plugged stainless steel tubes to allow for better circulation of helium during cooldown in order to avoid damage of the windows due to excessive pressure differences. These holes have negligible effect on the UCN storage lifetime defined further below. The superfluid ${ }^{4} \mathrm{He}$ is let in through a supply hole $(\varnothing 1 \mathrm{~cm})$ in the longer Al pipe (6). This provides good thermal contact of the UCN vessel, completely immersed in the superfluid, with a heat exchanger to a ${ }^{3} \mathrm{He}$ evaporation stage (7), via a $16 \mathrm{~cm}$ long column of superfluid ${ }^{4} \mathrm{He}$ in a " $U$ " tube (8) with inner diameter $1 \mathrm{~cm}$.

In the present experiments we used the UCN converter vessel with the majority of the inner surfaces coated with a thick layer (in the order of several tenths of $\mathrm{mm}$ ) of hydrogen-free perfluoropolyether grease (fomblin, Solvay Solexis RT15) with neutron optical potential $V_{\text {fomblin }}=$ $(115 \pm 10) \mathrm{neV}$. At low temperature the solidified fomblin layer cracks into many small flakes leading to a large surface and some holes to the substrate. Compared to the substrate materials the coating has, for neutrons with kinetic energy $E<V_{\text {fomblin }}$, a much smaller UCN loss rate per wall collision (characterised by a parameter $\eta$ with value $1.85 \times 10^{-5}$ for the fomblin used, see [31]). 


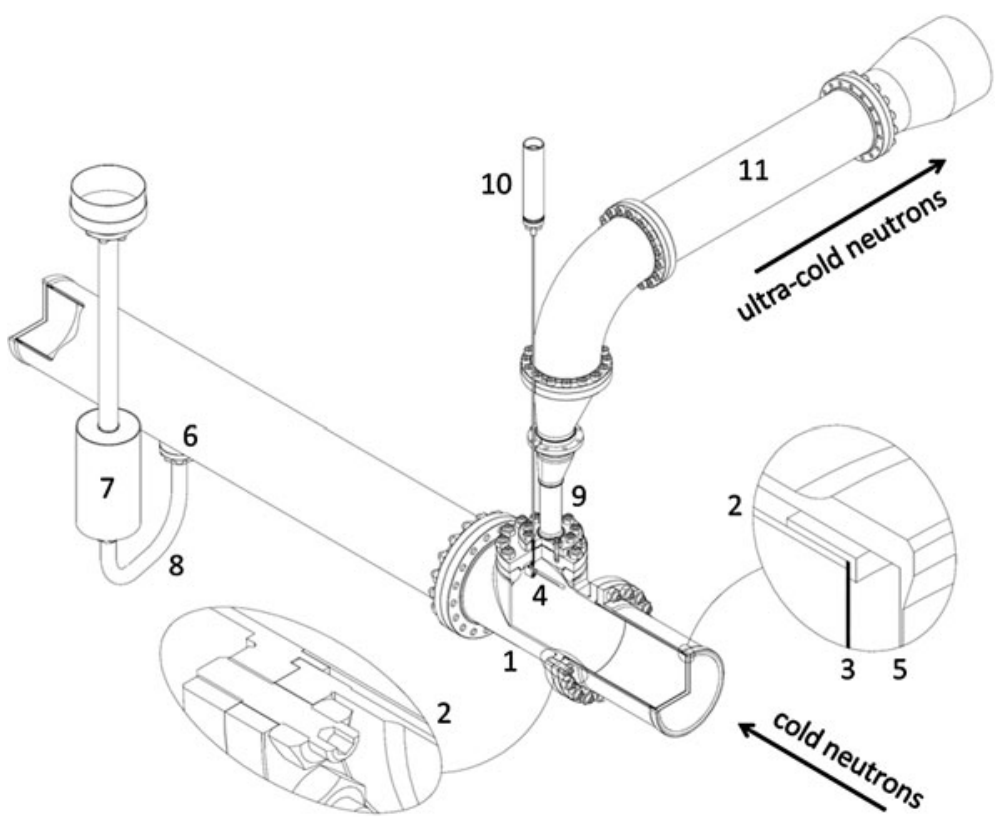

Fig. 1 Design drawing of the UCN converter vessel with the part of the UCN extraction channel which is situated in the isolation vacuum of the cryostat, partly in cut-away view. (1) "T" piece made of stainless steel, (2) stainless steel tube plugged in the "T" piece, (3) Ni window for cold neutron beam, (4) UCN flap valve, (5) Al window, (6) hole for supply of superfluid ${ }^{4} \mathrm{He}$ into the converter vessel, (7) heat exchanger between converter and a ${ }^{3} \mathrm{He}$ evaporation stage, (8) "U" tube, (9) UCN extraction chimney (thin-walled) followed by two tapered transitions

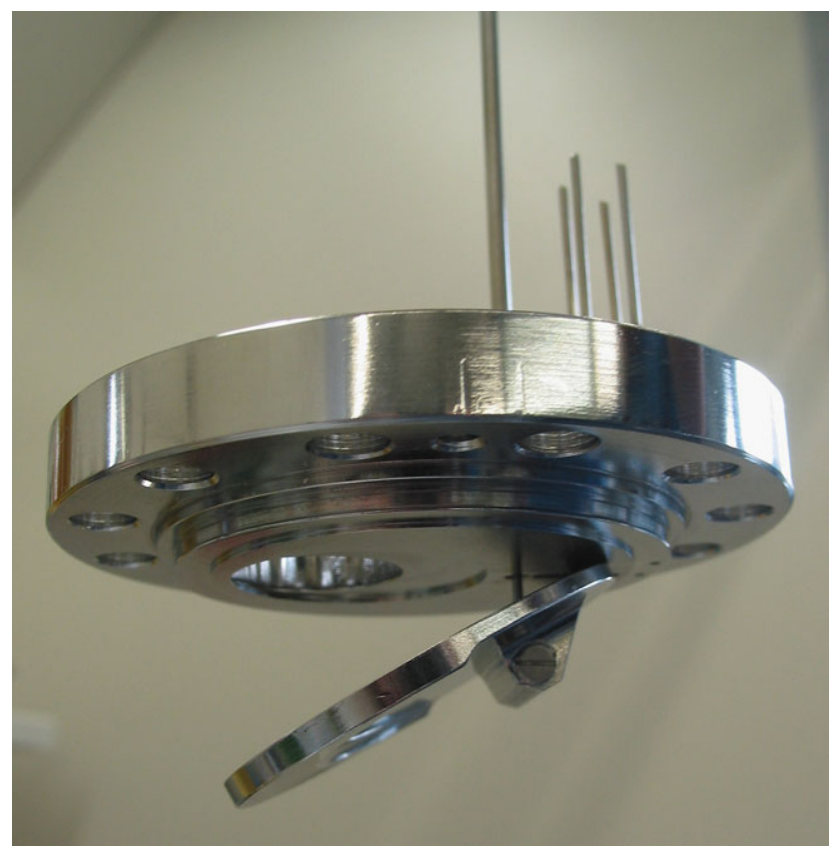

Fig. 2 Photograph of the open UCN flap valve. The vertical hole for UCN extraction can be closed by pulling the flap disk to the flange via a vertical actuator rod connected to a horizontal axis embedded in the disk, about which it is free to rotate. The flange is sealed with indium to the "T" section of the converter vessel (see Fig. 1) and a $90^{\circ}$ bend, (10) manipulator of UCN flap valve, (11) thin-walled stainless steel straight section of the UCN extraction guide, followed by a tapered transition connecting to the room-temperature neutron guide with inner diameter $66 \mathrm{~mm}$ (not shown). The detail magnifications show the arrangement of inner UCN vessel and outer jacket for confinement of the superfluid ${ }^{4} \mathrm{He}$. The longer pipe on the left side of the " $T$ " piece is made in the same way

Before the superfluid ${ }^{4} \mathrm{He}$ enters the vessel it is purified from ${ }^{3} \mathrm{He}$ by passage through a superleak (not shown in Fig. 1), consisting of a stainless steel tube with inner diameter $7 \mathrm{~mm}$ and wall thickness $0.5 \mathrm{~mm}$, filled with a $17 \mathrm{~cm}$ long column of compressed $\mathrm{Al}_{2} \mathrm{O}_{3}$ powder with grain size $50 \mathrm{~nm}$. For compactification of the powder we adopted the following process that takes about four hours. An Al block with a $15 \mathrm{~cm}$ deep hole is used to guide the tube vertically on a support. The tube is filled with a small quantity of powder through a funnel from paper and then the powder is hammered down for about one minute using a piston from steel. This process is repeated until the column of compressed powder has the desired length. After each step the filling height has on average increased by somewhat less than $1 \mathrm{~mm}$, thus requiring about 200 fillings. Finally the superleak is tightly soldered on the end for helium outlet into a wider tube, which provides an annular space with mean thickness $1 \mathrm{~mm}$ for radial cooling of the column of compressed powder along its whole length by the supplied superfluid. That way the superleak opens typically within several minutes once the helium at the entrance of the superleak has become superfluid.

The cold flap valve for UCN extraction (see Figs. 1 and 2) connects the converter vessel with electropolished stainless steel tubes which guide neutrons to a ${ }^{3} \mathrm{He}$-gas UCN detector at room temperature (for a description of the extraction 
guide system see [25]). The flap (4) is a disk of $4 \mathrm{~cm}$ diameter made of stainless steel. It was left uncoated in the present experiments to ensure safe operation when cooled down. To close the UCN extraction line (9) of $16 \mathrm{~mm}$ inner diameter, the disk can be pulled to the extraction aperture by means of a $1 \mathrm{~mm}$ thick stainless steel rod sliding inside a stainless steel capillary that was hard-soldered into the top flange of the " $T$ " section. A seal with a bellows at room temperature (10) enables operation of the valve using an air-pressurised cylinder with a spring.

In order to reduce the heat flow along the stainless steel extraction guide the two straight sections (9) and (11) were milled down to wall thickness $0.5 \mathrm{~mm}$. A thermal anchor between the two tapered transitions holds the end of the vertical section at a temperature below $10 \mathrm{~K}$, and a second anchor (not shown) situated in the horizontal straight section defines a point with a temperature of about $70 \mathrm{~K}$. Heat radiation along the extraction guide becomes noticeable when the flap valve is opened as a typical temperature increase of $20 \mathrm{mK}$.

The beam collimations used in the experiments described below were the same as described in [25]. Again, a neutron absorbing aperture of $33 \mathrm{~mm}$ diameter was placed at the entrance window to the converter, in order to avoid activation of the vessel during experiments with this prototype. Hence only a quarter of the total area of the entrance window to the vessel was irradiated with cold neutrons, with corresponding reduction of the total UCN production rate. Note however that we were interested only in determining the production rate density for a given flux density of the incident beam, rather than optimising the total number of UCN in this stage of development. Experiments were performed with the UCN converter vessel held at various temperatures down to $0.82 \mathrm{~K}$.

\section{Trapping potential and time constants}

The conversion process in superfluid ${ }^{4} \mathrm{He}$ produces a broad spectrum of low-energy neutrons in the converter vessel. The fate of these neutrons depends on their energy with respect to the wall potential of the vessel, the temperature of the converter, and on whether the UCN valve is open or closed. This section provides a framework for the analysis of the experiments described in subsequent sections.

The converter vessel provides a UCN trapping potential $V_{\text {trap }}$, defined by the difference of neutron optical potentials of the wall material and the neutron optical potential of the superfluid ${ }^{4} \mathrm{He}$ below $1 \mathrm{~K}\left(V_{4^{H e}}=18.5 \mathrm{neV}\right)$. For our vessel composed of several materials (fomblin, stainless steel and nickel), we employ the smallest neutron optical potential (i.e. that of the fomblin coating) in the definition of $V_{\text {trap }}$,

$V_{\text {trap }}=V_{\text {fomblin }}-V_{{ }^{4} \mathrm{He}}$.
Neutrons with kinetic energy $E<V_{\text {trap }}$ in the ${ }^{4} \mathrm{He}$ are trapped in the proper sense, i.e. propagating in the superfluid helium and being totally reflected by the surfaces of the converter vessel. Neutrons with energy within the range $V_{\text {trap }} \leq E<V_{\text {ss }}$ have some probability to enter the bulk of the fomblin coating but will still be reflected by the substrate. They might therefore survive some time in the vessel before being absorbed. However, as they spend a significant fraction of time in the bulk of the coating their chance to get lost is much higher than for the UCN having energies $E<V_{\text {trap }}$.

In order to investigate the typical time scale of this loss, Monte-Carlo simulations have been performed with Geant4UCN [33]. All experimental dimensions were modelled exactly. For the neutron optical potentials of stainless steel and fomblin the values stated in Sect. 2 were used. The fomblin coating was modelled as a homogenous layer with a roughness parameter 0.5 (meaning that $50 \%$ of the reflections are not specular) to account for the flake-like cracking of the coating at low temperatures. The stainless steel surface was modelled with a roughness parameter of 0.08 , which reproduces typical transmission values of $90 \% / \mathrm{m}$ for stainless steel guides. As a result of these simulations the abundance of neutrons with energies $V_{\text {trap }} \leq E<V_{\text {ss }}$ can safely be neglected with respect to the UCN when the full spectrum of neutrons is kept in the trap for more than 3 seconds. Hence, in the "buildup-mode measurements" described in Sect. 4 these neutrons can usually be neglected but in the "continuous-mode measurements" described in Sect. 6 they may have to be considered.

Note that also the spectrum of truly trapped UCN with kinetic energies $E<V_{\text {trap }}$ becomes shaped due to energydependent wall losses. Both the loss probability per wall collision and the frequency of wall collisions increase with $E$, giving those UCN with energies close to $V_{\text {trap }}$ the largest probability to get lost (the formulas describing energydependent losses of UCN on wall collisions can be found in the books $[1,2])$.

Storage and extraction of UCN from the converter can be characterised by various parameters. The storage lifetime $\tau$ quantifies the temporal decrease of UCN in the closed vessel. The rate constant

$\tau^{-1}(E, T)=\tau_{0}^{-1}(E)+\tau_{\text {up }}^{-1}(T)+\tau_{\beta}^{-1}$

contains: (1) a neutron energy-dependent contribution $\tau_{0}^{-1}(E)$ due to losses on wall collisions or through holes, and due to absorption by impurities in the converter bulk, (2) a contribution due to temperature-dependent UCN upscattering, and (3) a rate constant for neutron beta decay.

A second parameter is the emptying time constant $\tau_{\mathrm{e}}$ which quantifies the temporal decrease of UCN in the vessel with the UCN valve open and with the cold beam switched 
off:

$\tau_{\mathrm{e}}^{-1}(E, T)=\tau^{-1}(E, T)+\tau_{A}^{-1}(E)$.

The time constant $\tau_{A}$ characterises UCN passage through the exit hole with area $A$ and the extraction tubing.

From these time constants we may derive the probability to detect a UCN present in the converter vessel (while the extraction valve is kept open to give it a chance to reach the detector). It is given by

$W(E, T)=\varepsilon(E) \frac{\tau(E, T)-\tau_{\mathrm{e}}(E, T)}{\tau(E, T)}$,

and characterises the efficiency of the whole system including extraction. The factor $0<\varepsilon(E)<1$ describes losses due to imperfections in extraction and detection.

An analysis of the measurements described below within this framework has to take into account the neutron energy dependence of the quantities involved. In the present experiments (like in the previous ones published in [25]) we did not detect the UCN as a function of energy. Therefore we are interested in working, whenever possible, in terms of ensemble average values of the various parameters. If applicable we denote them by the same symbols as in (2), (3) and (4), omitting the respective arguments $E$ and $T$.

\section{Experiments on UCN accumulation}

In the "buildup mode" experiments described here the closed converter is first irradiated with cold neutrons for a period of UCN accumulation $t_{0}$, after which the beam is shut off and simultaneously the UCN valve is opened. During the whole process a time histogram of the UCN count rate is recorded. Figure 3 shows a series of such histograms for different $t_{0}$, obtained with the fomblin-coated converter.

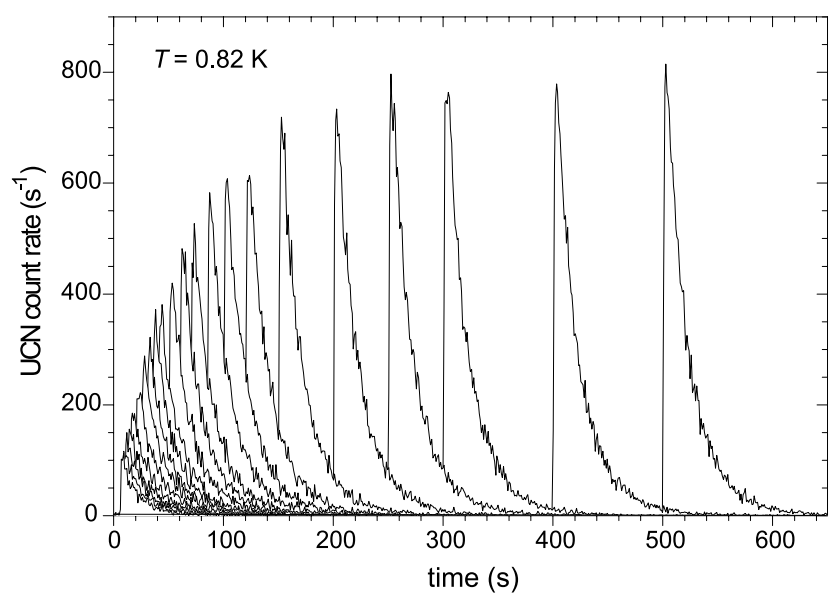

Fig. 3 Time histograms of UCN count rates, measured in buildup mode at $0.82 \mathrm{~K}$ for 23 different accumulation times $t_{0}$
As first observation we find that the temporal decrease of the neutron count rate after opening the $\mathrm{UCN}$ valve is perfectly described by a single-exponential fit of $A \exp (-t / \tilde{\tau})$ to the data, thus providing for each histogram a unique, ensemble average value $\tilde{\tau}$, which we identify with $\tilde{\tau}=\tau_{\mathrm{e}}$. However, when looking at the dependence of the values of $\tau_{\mathrm{e}}$ on the UCN accumulation time $t_{0}$, the influence of spectral shaping of the trapped neutrons becomes visible. Figure 4 shows that, with decreasing converter temperature, $\tau_{\mathrm{e}}$ tends to increase for longer $t_{0}$. As the relative weight of the wall-induced contribution in (2) is largest at the lowest temperature, spectral shaping effects might ideally become negligible at sufficiently high temperature, where the influence of $\tau_{\text {up }}^{-1}(T)$ becomes dominant. At the highest temperature investigated, $T=1.26 \mathrm{~K}$, the values of $\tau_{\mathrm{e}}$ are indeed found to be almost $t_{0}$-independent, with value $\tau_{\mathrm{e}}=(9.92 \pm 0.15) \mathrm{s}$. Still, with a reduced $\chi^{2}=2.3$ for 9 degrees of freedom (DOF) the fit of a constant value to the data is not perfect.

Values for storage lifetimes $\tau$ can be obtained from the dependence of UCN count rates on the accumulation time $t_{0}$. In Fig. 5 we plotted the integrals $N\left(t_{0}\right)$ of all histograms measured in the buildup mode. In first place the data demonstrate the saturation behaviour of UCN accumulation and a strong gain of UCN output for the lower temperatures within the $T$ range investigated. We analysed the data for the various temperatures using the following single-exponential buildup function:

$N\left(t_{0}\right)=N_{\infty}\left(1-\exp \left(-\frac{t_{0}+\Delta t}{\tau}\right)\right)+N_{\mathrm{b}}$.

$N_{\infty}$ is the number of UCN in saturation, and $N_{\mathrm{b}}$ is introduced to account for some effect discussed below. A systematic offset $\Delta t$ on the nominal accumulation time $t_{0}$ is

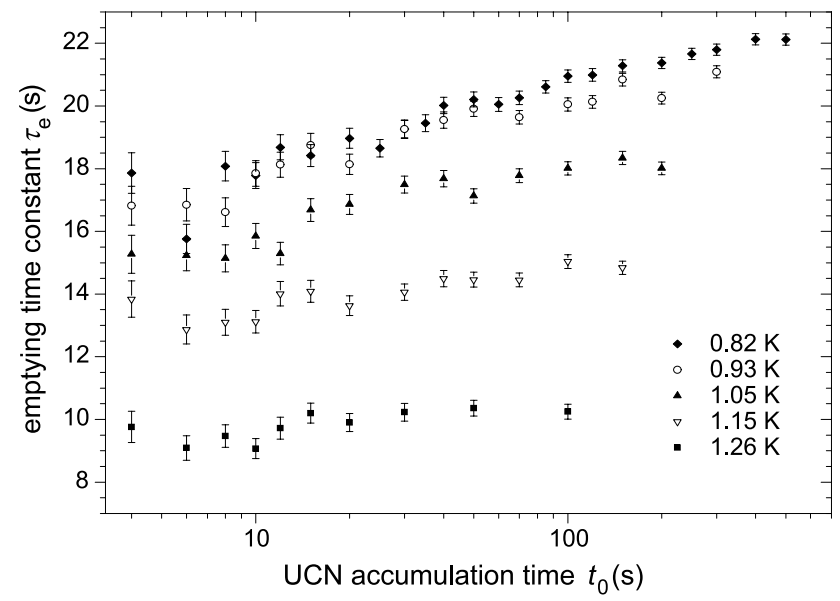

Fig. 4 Emptying time constants $\tau_{\mathrm{e}}$ as a function of the UCN accumulation time $t_{0}$, determined from the histograms of all buildup-mode measurements for the various converter temperatures. Error bars show uncertainties obtained from the corresponding fits to the data 
due to different delays when opening and closing the coldneutron beam shutter. It was determined in a separate experiment where we mounted behind the shutter a horizontal, neutron absorbing slit in the center of the beam. Behind the slit we installed a neutron detector and measured the delay with which the heavy shutter block opened or closed the beam passing the slit, after having sent an electronic signal to open or close the shutter. We thus found delay times of $0.81 \mathrm{~s}$ for opening, and 1.13 for closing the beam. Hence, $\Delta t=0.32 \mathrm{~s}$, independent of the setting of $t_{0}$.

Table 1 shows the results of fits to our complete set of data. For the highest temperature (5) provides an adequate description of UCN accumulation. The fit becomes worse with lower temperatures, where longer storage lifetimes can be expected to lead to more pronounced spectral shaping. The values for $N_{\mathrm{b}}$ contain an obvious contribution due to the delay of the shutter described before. While it takes about a second to close the cold beam after having sent an electronic signal, the UCN valve opens with a delay not exceeding two tenths of a second. We should therefore expect this contribution to be given by the product of one second and the rate $\dot{N}_{\mathrm{c}}$

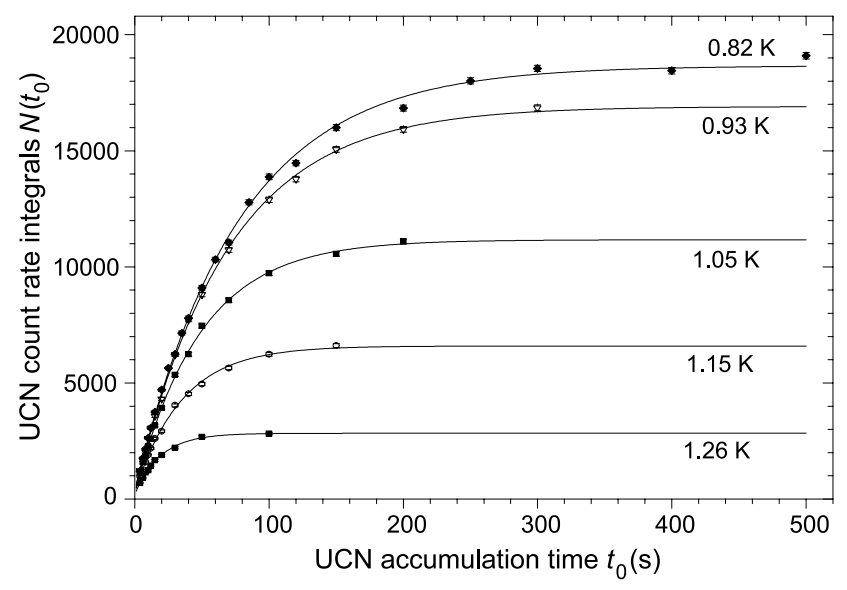

Fig. 5 UCN count rate integrals, measured in buildup mode for the various temperatures as a function of the accumulation time $t_{0}$. The lines show fits of the single-exponential buildup function defined in (5), with the parameters quoted in Table 1

Table 1 Fit results using our complete set of data. Values for $\tau, N_{\infty}$, and $N_{\mathrm{b}}$ were determined using (5) from the count rate integrals in buildup mode, shown in Fig. 5. The values for $\tau_{\mathrm{e}}$ are mean values of the data shown in Fig. 4. Using (3) they provide values for $\tau_{A}$. Values of neutrons as measured in the steady state with the UCN valve open (see Sect. 6). The numerical values of $N_{\mathrm{b}}$ from Table 1 and $\dot{N}_{\mathrm{c}}$ quoted in Table 2 are indeed so similar that $N_{\mathrm{b}}$ seems to be fully accounted for by this effect and does not contain any other contribution.

\section{$5 \mathrm{UCN}$ accumulation with delayed extraction}

Experiments with delayed UCN extraction are a variant of the buildup-mode measurements described in the previous section. Again the converter is first irradiated with cold neutrons for an accumulation period $t_{0}$ while the UCN valve stays closed, but the UCN valve is now opened with a delay time $t_{\mathrm{d}}$ after closing the beam.

Such experiments provide values for $\tau$ as a function of accumulation time $t_{0}$ and delay time $t_{\mathrm{d}}$. Figure 6 shows a plot of the count rate integrals of two series of measurements, for $t_{0}=25 \mathrm{~s}$ and for $t_{0}=250 \mathrm{~s}$, both at $0.82 \mathrm{~K}$. Values for $\tau$ can be obtained from single-exponential fits to a group of several data points. For these fits one selects a range

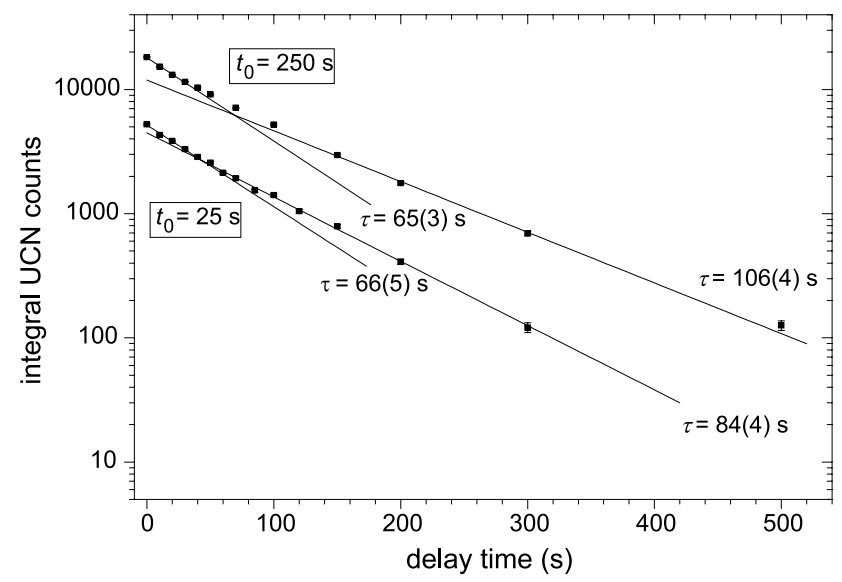

Fig. 6 Count rate integrals of the measurements at $0.82 \mathrm{~K}$ with delayed extraction after UCN accumulation times $t_{0}=25 \mathrm{~s}$ (lower data points) and $250 \mathrm{~s}$ (upper data points). The solid lines are fits of single exponentials to the four first, respectively, four last data points. The corresponding time constants $\tau$ derived from these fits are also shown

for $W / \varepsilon$ are deduced using (4). The observed systematic worsening of fits with decreasing temperature may be expected due to spectral shaping effects. All uncertainties were scaled to provide fits with $\chi^{2}=1$

\begin{tabular}{llllllll}
\hline$T[\mathrm{~K}]$ & $\tau[\mathrm{s}]$ & $\chi^{2}(\mathrm{DOF})$ & $\tau_{\mathrm{e}}[\mathrm{s}]$ & $\tau_{A}[\mathrm{~s}]$ & $N_{\mathrm{b}}$ & $N_{\infty}$ & $W / \varepsilon[\%]$ \\
\hline 1.26 & $19.3 \pm 1$ & $0.91(8)$ & $9.92 \pm 0.15$ & $20.4 \pm 1.3$ & $167 \pm 42$ & $2666 \pm 48$ & $48.6 \pm 2.8$ \\
1.15 & $35.2 \pm 1.6$ & $2.5(11)$ & $14.3 \pm 0.17$ & $24.1 \pm 0.89$ & $253 \pm 57$ & $6339 \pm 98$ & $59.4 \pm 1.9$ \\
1.05 & $48.6 \pm 1.2$ & $1.6(12)$ & $17.4 \pm 0.26$ & $27.1 \pm 0.73$ & $174 \pm 43$ & $10994 \pm 99$ & $64.2 \pm 1.0$ \\
0.93 & $69.5 \pm 1.4$ & $2.1(14)$ & $19.8 \pm 0.28$ & $27.7 \pm 0.59$ & $232 \pm 44$ & $16678 \pm 133$ & $71.5 \pm 0.7$ \\
0.82 & $76.5 \pm 1.4$ & $3.7(21)$ & $20.7 \pm 0.27$ & $28.4 \pm 0.54$ & $291 \pm 52$ & $18372 \pm 128$ & $72.9 \pm 0.8$ \\
\hline
\end{tabular}


of delay times during which the slope of a smooth curve through the data points shown in Fig. 6 does not change significantly due to spectral shaping. The values obtained for $\tau$ thus represent the apparent storage lifetime of the ensemble of UCN remaining in the vessel after a mean delay time $t_{\mathrm{d}}$. Values for this quantity that are least affected by spectral shaping can be obtained if one takes only data of two adjacent times $t_{\mathrm{d}_{1}}$ and $t_{\mathrm{d}_{2}}$, using the relation

$\tau=\frac{t_{\mathrm{d}_{2}}-t_{\mathrm{d}_{1}}}{\ln N\left(t_{\mathrm{d}_{1}}\right)-\ln N\left(t_{\mathrm{d}_{2}}\right)}$.

Data points should however not be chosen too close together in time as then the method becomes insensitive, leading to large error bars. This is visible for the short delay times in Fig. 7, which shows the values of $\tau$ obtained from (6) for the two series. The variation of $\tau$ from one to two minutes for $t_{0}=250 \mathrm{~s}$ demonstrates the spectral shaping of the UCN during the delay time $t_{\mathrm{d}}$ due to the energy-dependent wall collisions.

Comparing the two sets of data in Fig. 7, for short delay times no significant influence of $\tau$ on $t_{0}$ is noticeable within the accuracy of measurements. Only after long $t_{\mathrm{d}}$ the values of $\tau$ for $t_{0}=25 \mathrm{~s}$ tend to be lower than those for $t_{0}=250 \mathrm{~s}$. These findings might be expected, as after a longer accumulation time the relative abundance of slow UCN due to their longer lifetime becomes larger than for a short $t_{0}$. For short delay times, however, their effect on the storage lifetime is still masked by the more abundant faster neutrons.

The presence of neutrons with long storage lifetime in the vessel obviously can be made visible very well in the delayed-extraction experiments by waiting long enough until the faster neutrons will have "died away", whereas in the buildup-mode experiments "fresh" UCN are continuously

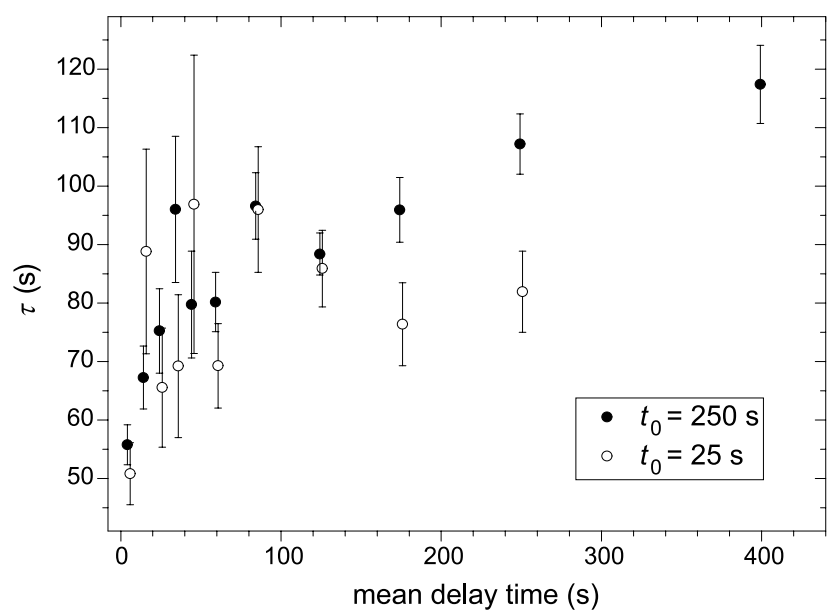

Fig. 7 UCN storage time constants deduced from (6), using adjacent data points from the measurements with delayed extraction shown in Fig. 6. The abscissa is given by the mean of the two adjacent delay times, $\left(t_{\mathrm{d}_{i+1}}+t_{\mathrm{d}_{i}}\right) / 2$ refurbished with the full spectrum, and therefore the variations of $\tau$ seem weaker.

\section{Experiments with open converter vessel}

In the "continuous-mode" experiments described here we determined a steady count rate $\dot{N}_{\mathrm{c}}$, measured with the converter irradiated for a long time with cold neutrons while the UCN valve stayed open. Figure 8 shows data from such measurements, performed at four different temperatures.

For the higher temperatures the count rates in the flat intensity regions slightly decrease with time. This is due to warming of the helium bath resulting from thermal radiation entering the vessel when the UCN valve is kept open. For closed valve this radiation is reflected back into the extraction guide. Whereas the effect of the temperature increase of typically $20 \mathrm{mK}$ on the neutron count rates is well visible at high temperature it is not noticeable for the lowest temperature. Table 2 quotes values of plateau count rates $\dot{N}_{\mathrm{c}}$ for all temperatures at which measurements were performed. The data show indeed that count rates change only little at low temperature, where $|\mathrm{d} W / \mathrm{d} T|$ becomes small (as can be estimated from the values in Table 1) because UCN

Table $2 T$-dependent count rates $\dot{N}_{\mathrm{c}}$ obtained from continuous-mode measurements. The values are deduced from the intensity plateaus, which in Fig. 8 are shown for four of the five temperatures

\begin{tabular}{llllll}
\hline$T[\mathrm{~K}]$ & 1.26 & 1.15 & 1.05 & 0.93 & 0.82 \\
\hline$\dot{N}_{\mathrm{c}}\left[\mathrm{s}^{-1}\right]$ & $176 \pm 3$ & $228 \pm 3$ & $258 \pm 2$ & $278 \pm 2$ & $287 \pm 2$ \\
\hline
\end{tabular}

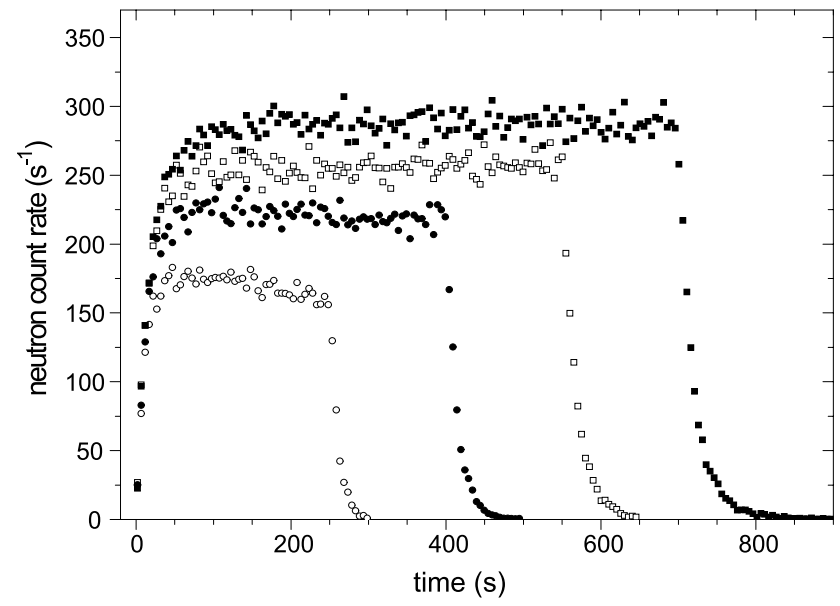

Fig. 8 Count rates measured in continuous mode for four temperatures: $T=1.26 \mathrm{~K}$ (open circles), $1.15 \mathrm{~K}$ (full circles), $1.05 \mathrm{~K}$ (open squares), and $0.82 \mathrm{~K}$ (full squares). The cold neutron beam shutter was opened at time $t=0$, and closed after $250 \mathrm{~s}, 400 \mathrm{~s}, 550 \mathrm{~s}$, and $700 \mathrm{~s}$. The flat regions were used to determine $\dot{N}_{\mathrm{c}}$ for the corresponding temperatures 


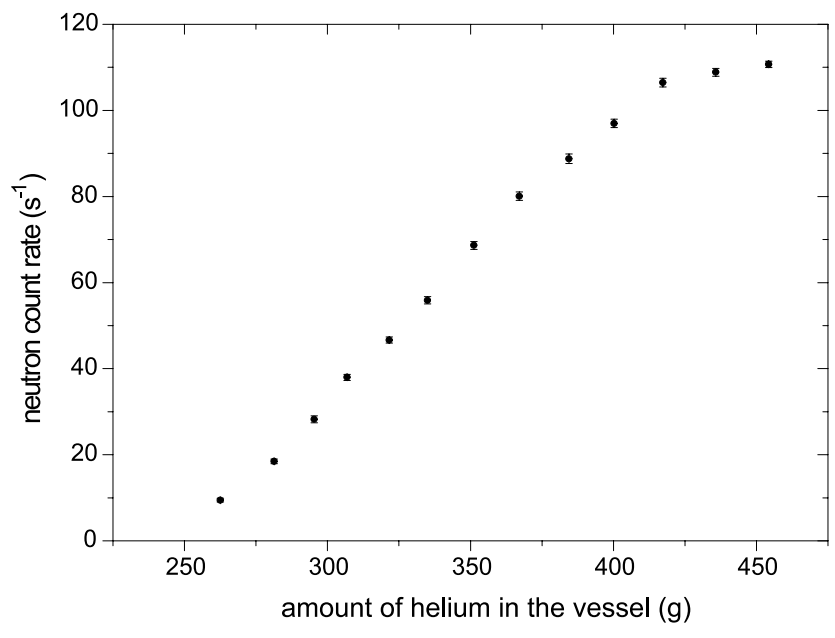

Fig. 9 Count rate $\dot{N}_{\mathrm{c}}$ measured during filling the UCN converter vessel with superfluid helium at $1.44 \mathrm{~K}$, demonstrating that one may apply the rate of UCN production as a "helium level meter"

escape through the open flap valve then dominates over upscattering.

The continuous-mode measurements can also serve as a "level meter" for the superfluid helium. Figure 9 shows repeated measurements of $\dot{N}_{\mathrm{c}}$ during filling the vessel. The net ${ }^{4} \mathrm{He}$ mass flow into the vessel was determined by means of two flow meters. Since our cryostat liquefies the helium for the converter from a gas bottle, we could easily measure the incident flow. A second flow meter behind a pump connected to the vessel measured the evaporation rate from the helium bath. The abscissa of Fig. 9 is the difference of both flows, integrated over time. During filling, the temperature of the vessel could be stabilised to $1.44 \mathrm{~K}$. The shape of the curve is as expected for the helium level rising behind the circular entrance aperture for the cold neutron beam.

\section{Incident cold neutron beam spectrum}

For determination of the UCN production rate density one needs to know the intensity of the incident cold neutron beam as a function of the neutron wavelength. We measured this spectrum in a separate experiment, using a time-of-flight (TOF) setup consisting of a mechanical chopper and a ${ }^{3} \mathrm{He}$ detector with a vertical slit. Details of the apparatus are described in [32].

For this measurement the apparatus for UCN production was removed and the entrance aperture of the TOF analyser was placed at the former position of the entrance window to the converter vessel. The detector was moved horizontally during measurements in order to integrate over the whole divergence of the beam. We thus determined the neutron wavelength spectrum up to $\lambda=2 \mathrm{~nm}$ (frame overlap of the chopped bunches occured only for $\lambda>5 \mathrm{~nm}$, where the intensity is negligibly small). Figure 10 shows the spectrum in

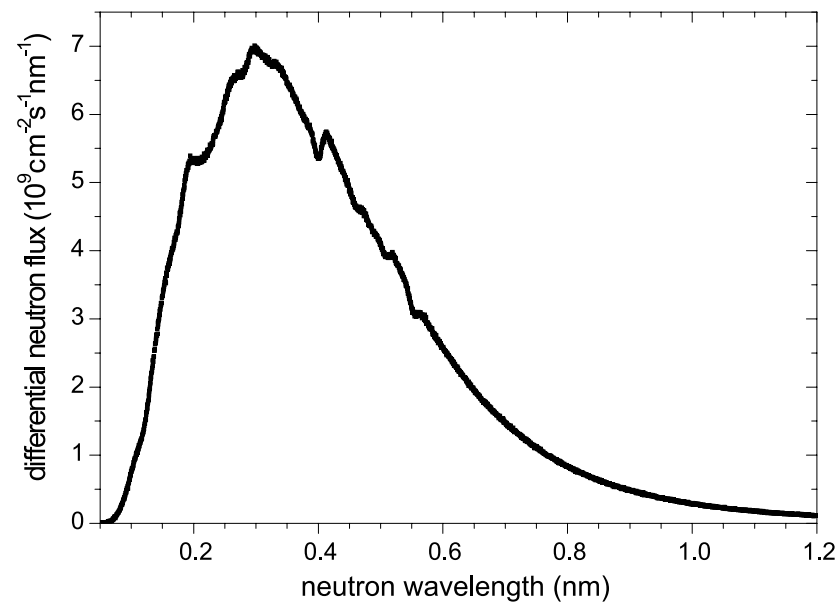

Fig. 10 Differential flux $\mathrm{d} \phi / \mathrm{d} \lambda$ of the neutron beam used for the study of UCN production, measured by time-of-flight at the neutron guide NL1 of FRM II (note that the untypical position of the intensity maximum at only $0.3 \mathrm{~nm}$ is due to incomplete filling of the cold $\mathrm{D}_{2}$ source during the reactor cycle in which these experiments were performed)

the range $\lambda \leq 1.2 \mathrm{~nm}$. After the TOF measurements the integral neutron flux was determined with a gold foil activation in order to normalise the spectrum.

Relevant for UCN production via emission of a single phonon is the differential flux at $\lambda^{*}=0.89 \mathrm{~nm}$, for which we thus obtained

$\mathrm{d} \phi /\left.\mathrm{d} \lambda\right|_{\lambda^{*}}=5.0 \times 10^{8} \mathrm{~cm}^{-2} \mathrm{~s}^{-1} \mathrm{~nm}^{-1}$.

The differential flux for neutron wavelengths shorter than $\lambda^{*}$ is required to calculate UCN production via multi-phonon processes. The determination of the UCN production rate using the knowledge of the neutron wavelength spectrum provided by the TOF measurement is discussed in the next section.

\section{UCN production rate}

We determine the UCN production rate using two methods. Both rely on the fact that after long irradiation with cold neutrons the rates for UCN production and escape from the vessel become equal.

First, as in the analysis of our last experiments [25], we use the stationary count rate $\dot{N}_{\mathrm{c}}$ from the continuous-mode measurements. The condition of equal rates, for UCN production on one hand, and loss in the vessel or escape through the UCN extraction guide on the other, leads us to the following expression for the corresponding UCN production rate density:

$P_{1}=\frac{\dot{N}_{\mathrm{c}}}{V_{\mathrm{p}} W}$ 
Table 3 Production rate densities, deduced from the data quoted in Tables 1 and 2. Only counting statistical uncertainties are quoted

\begin{tabular}{llllll}
\hline$T[\mathrm{~K}]$ & 1.26 & 1.15 & 1.05 & 0.93 & 0.82 \\
\hline$\varepsilon P_{1}\left[\mathrm{~s}^{-1} \mathrm{~cm}^{-3}\right]$ & $0.61 \pm 0.04$ & $0.64 \pm 0.02$ & $0.67 \pm 0.01$ & $0.65 \pm 0.01$ & $0.66 \pm 0.01$ \\
$\varepsilon P_{2}\left[\mathrm{~s}^{-1} \mathrm{~cm}^{-3}\right]$ & $0.48 \pm 0.04$ & $0.51 \pm 0.03$ & $0.59 \pm 0.02$ & $0.56 \pm 0.01$ & $0.55 \pm 0.01$ \\
\hline
\end{tabular}

For $\dot{N}_{\mathrm{c}}$ and $W / \varepsilon$ we take the values stated in Tables 1 and 2 . The UCN production volume $V_{\mathrm{p}}=595 \mathrm{~cm}^{3}$ is given by the product of length of the vessel and the beam area defined by the entrance aperture. ${ }^{1}$

A second method employs the saturation behaviour of the buildup-mode measurements. Corresponding values $P_{2}$ may be derived from the parameter $N_{\infty}$ in (5). Denoting with $N_{0}$ the saturated number of UCN in the vessel, of which only the fraction $N_{\infty}=N_{0} W$ is detected, this leads us to

$P_{2}=\frac{N_{\infty}}{W V_{\mathrm{p}} \tau}$.

For evaluation we use the values of the respective quantities from Table 1, with $V_{\mathrm{p}}$ as before.

The results from both methods are quoted in Table 3 . The comparison shows that the values for $P_{1}$ are systematically larger than those for $P_{2}$. This can be understood by noting that we cannot exclude contamination of the value $\dot{N}_{\mathrm{c}}$ due to neutrons with energies $E>V_{\text {trap }}$. As the flap valve is kept open during cold-neutron irradiation, some of these neutrons with storage lifetime much shorter than $\tau$ may find the exit hole after only a few wall collisions. The value $P_{1}$ might therefore over-estimate the true UCN production rate density.

In the Monte Carlo simulations, where the roughness parameter of the extraction guide system was varied, it was indeed found that $P_{1}$ might be up to $30 \%$ too high due to neutrons with $E>V_{\text {trap. }}$. For larger roughness in the extraction guide simulated values became slightly reduced, whereas a variation of the surface roughness of the fomblin coating had no significant influence. In the determination of $P_{2}$ on the other hand we expect this overestimation of the true UCN production rate density to be negligible, as neutrons with $E>V_{\text {trap }}$ are already taken into account by $N_{\mathrm{b}}$.

The data taken at a temperature of $1.26 \mathrm{~K}$ are well described with the single-exponential buildup function given in (5), with reduced $\chi^{2}=0.91$ of the fit (see Table 1 ). We therefore assume that in this measurement spectral shaping is negligible for our analysis. The experimental value

$\varepsilon P_{\exp }=(0.48 \pm 0.04) \mathrm{s}^{-1} \mathrm{~cm}^{-3}$

\footnotetext{
${ }^{1}$ Note that the incident beam slightly diverges throughout the vessel, hence the value stated in (8) gives the UCN production rate density close to the entrance aperture. If the vessel includes a guide for cold neutrons (as planned for a later upgrade of our apparatus), the divergence of a beam irradiating the entire cross section of the guide will not reduce the flux density.
}

thus seems a conservative estimate of the true UCN production rate density.

We may now compare the experimental findings with the theoretical expectation, up to the unknown factor $\varepsilon$. The mechanism of UCN production contains contributions from emission of a single phonon and multi-phonon processes. The single-phonon production rate density in a helium converter with Be wall coating is $P_{\mathrm{I}}=(4.97 \pm$

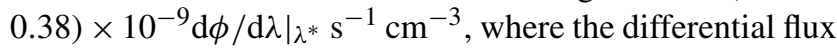
at $\lambda^{*}=0.89 \mathrm{~nm}$ is given in $\mathrm{cm}^{-2} \mathrm{~s}^{-1} \mathrm{~nm}^{-1}$ [24]. For the present situation this value needs to be corrected for the neutron optical potential of the fomblin grease, $V_{\text {fomblin }}$, i.e. divided by $(252-18.5)^{3 / 2} /(115-18.5)^{3 / 2}=3.76$, with an uncertainty of 0.59 due to the poor knowledge of $V_{\text {fomblin }}$. From the measured neutron TOF spectrum with the value of differential flux stated in (7) we expect

$P_{\mathrm{I}}=(0.66 \pm 0.11) \mathrm{s}^{-1} \mathrm{~cm}^{-3}$.

The differential multi-phonon production rate density is given by

$\frac{\mathrm{d} P_{\mathrm{II}}}{\mathrm{d} \lambda}=\frac{\sqrt{2 m_{\mathrm{n}}}}{3 \pi \hbar} V_{\text {trap }}^{3 / 2} n_{4{ }_{\mathrm{He}}{ }^{{ }^{4} \mathrm{He}}} \frac{\mathrm{d} \phi}{\mathrm{d} \lambda} s_{\mathrm{II}}(\lambda) \lambda$.

In this expression enters the number density of the helium atoms, $n_{4} \mathrm{He}=2.1835 \times 10^{22} \mathrm{~cm}^{-3}$, and the neutron (bound) scattering cross section per helium atom, $\sigma_{4} \mathrm{He}=1.34 \times$ $10^{-24} \mathrm{~cm}^{2}$. The quantity $s_{\mathrm{II}}(\lambda)$ is the scattering function $s_{\mathrm{II}}(Q, \omega)$, evaluated on the dispersion curve of the free neutron, i.e. $\omega=\hbar Q^{2} /\left(2 m_{\mathrm{n}}\right)$, with $Q=2 \pi / \lambda$. It is modelled, see [24], to fit inelastic neutron scattering data from [34]. Integration of $\mathrm{d} P_{\mathrm{II}} / \mathrm{d} \lambda$ from $0.35 \mathrm{~nm}$ to $\infty$ for the measured incident differential flux provides

$P_{\mathrm{II}}=(0.62 \pm 0.09) \mathrm{s}^{-1} \mathrm{~cm}^{-3}$

for the integral multi-phonon production rate.

The main uncertainty comes from contributions to $P_{\mathrm{II}}$ in the wavelength range $\lambda<0.52 \mathrm{~nm}$. First, there is no direct experimental information available about $s_{\mathrm{II}}(\lambda)$, and second, there is a strong weight due to the maximum of $\mathrm{d} \phi / \mathrm{d} \lambda$ in this region (see Fig. 10). The $\lambda$-dependent measurements of UCN production by Baker and colleagues [21] indicate that a multi-phonon contribution exists also from the region $0.4 \mathrm{~nm} \leq \lambda \leq 0.52 \mathrm{~nm}$. The measured integral multi-phonon and single-phonon production rates were found to add up evenly to the measured integral production rate [35]. This provides an experimental hint that for $\lambda<0.4 \mathrm{~nm}$ the multiphonon contribution might be negligible. Therefore we expect the total UCN production rate density to be given by 
the sum of values stated in (11) and (13),

$P_{\mathrm{th}}=(1.28 \pm 0.14) \mathrm{s}^{-1} \mathrm{~cm}^{-3}$.

Additional experimental information about $s_{\mathrm{II}}(\lambda)$ will be required for a more accurate prediction of the UCN production rate induced by a white cold neutron beam. Note that this is particularly necessary for the region of short wavelengths, whereas the contribution to $P_{\text {II }}$ from the region $\lambda>0.8 \mathrm{~nm}$ is only $0.03 \mathrm{~s}^{-1} \mathrm{~cm}^{-3}$.

\section{Discussion and conclusions}

Our experiments with the present prototype represent first steps towards an intense UCN source for user applications, employing superfluid ${ }^{4} \mathrm{He}$ at a cold neutron beam. This validates an old idea [23] which has long awaited to become converted into a versatile tool. Twenty years ago in a first attempt to accumulate and extract UCN from superfluid ${ }^{4} \mathrm{He}$ only a small fraction of the UCN could be observed outside the cryostat [26]. As a result, nowadays several projects employ the converter in-situ, thus avoiding UCN extraction from the helium. With the vertical, window-less extraction of UCN an efficient scheme has been found which will widen the scope of this type of converter, enabling experiments to be performed also at room temperature.

In the present experiments we have characterised the performance of a fomblin-coated converter vessel and measured the temperature dependence of UCN production. With an analysis of the results in terms of ensemble-averaged quantities we could determine various time constants characterising UCN storage loss rates and UCN extraction speed from the converter vessel. Compared to our previous, first experiment with the uncoated electropolished stainless steel converter vessel with $\mathrm{Ni}$ windows [25], count rates detected in comparable situations were about a factor three higher, despite the lower neutron optical potential of the fomblin coating. The increased UCN output is due to longer storage time constants of the closed vessel and smaller time constants for escape of UCN through the open valve.

Values for $\tau_{A}$ as derived from (3) can be compared with a prediction from the gas-kinetic equation

$\tau_{A}=\frac{4 V}{v A}$,

where we insert $V \approx 2.41$ for the volume of the converter vessel, and the mean velocity $v$ of the trapped UCN. For a uniform distribution in velocity space, $v=3 v_{\max } / 4$ with $m_{\mathrm{n}} v_{\max }^{2} / 2=V_{\text {wall }}-V_{4} \mathrm{He}$, where $m_{\mathrm{n}}$ is the neutron mass. For the fomblin-coated vessel $\left(V_{\text {wall }}=V_{\text {fomblin }}\right)$, we thus may expect $\tau_{A}=14.8 \mathrm{~s}$. The mean value from all data measured at $T=1.26 \mathrm{~K}$ was $\tau_{A}=(20.4 \pm 1.3) \mathrm{s}$. In our earlier experiments with the uncoated stainless steel converter vessel $\left(V_{\text {wall }}=V_{\mathrm{ss}}\right)$, we may expect $\tau_{A}=11.3 \mathrm{~s}$ but measured $\tau_{A}=(58 \pm 13) \mathrm{s}$. The large discrepancy might be due to UCN trajectories not exploring quickly enough the available phase space in the electropolished vessel, a hypothesis already raised earlier by W. Mampe and co-workers for their liquid-wall UCN bottle experiment [36]. Best results might be obtained when the majority of the vessel walls will be realised as mirrors in a future source implementation, and roughness be introduced only in some extended region close to the extraction hole. The mirrors would not only guide the cold neutron beam but also allow for specular UCN transport towards the extraction hole where diffuse scattering of neutrons out of quasi-stable orbits should increase their extraction probability. In addition it should be avoided that the flap valve, while open, acts as an obstacle.

UCN production rates could be derived from experiments performed at the highest temperature, where so far in all our studies spectral shaping effects seem to be negligible. The values obtained in our earlier experiment, $\varepsilon P_{1}=$ $(0.91 \pm 0.21) \mathrm{s}^{-1} \mathrm{~cm}^{-3}$, and $\varepsilon P_{2}=(1.09 \pm 0.25) \mathrm{s}^{-1} \mathrm{~cm}^{-3}$, were higher due to the higher neutron optical potential of stainless steel. One may also expect $\varepsilon$ for the corresponding $\mathrm{UCN}$ energy spectrum to be higher, since UCN with low energy should have a lower probability to overcome the gravitational barrier of the extraction guide, and also the detector has a somewhat lower efficiency for these neutrons.

In the present study we could not only reduce significantly the counting statistical uncertainties but we also performed a complementary TOF measurement of the incident beam. This allows us now to draw conclusions about the UCN extraction and detection efficiency. The TOF measurement provides the wavelength-differential neutron flux of the incident beam, which scales the strength of the singleand multi-phonon UCN production rates. The deduced value $P_{\text {th }}$ provides a benchmark for comparison with experimental values $\varepsilon P_{\exp }$ derived from measured time constants and UCN count rates. The factor $\varepsilon$ accounts for the combined efficiency of extraction guide and detector. Assuming validity of the theory of $\mathrm{UCN}$ production, i.e. $P_{\exp }=P_{\text {th }}$, we can determine $\varepsilon$ from the ratio $\varepsilon P_{\exp } / P_{\text {th }}$.

The overall efficiency $W$ of the whole system consisting of converter vessel, extraction guide and detector, was given in (4). Noting that from the time constants we could only determine ( $\varepsilon$-independent) experimental values for $W / \varepsilon$ (see Table 1), we may now express $W$ in terms of the product of two ratios,

$W=\frac{\varepsilon P_{\exp }}{P_{\mathrm{th}}} \frac{\tau-\tau_{\mathrm{e}}}{\tau}$.

Using the most conservative value for the UCN production rate density obtained in the present analysis as stated in (10), we find from the first ratio in (16) a value of $\varepsilon=38 \%$. Since $\varepsilon$ may be expressed as product $\varepsilon=\varepsilon_{\mathrm{ex}} \varepsilon_{\mathrm{det}}$ of extraction and detection efficiencies, and we cannot expect 
$\varepsilon_{\text {det }}$ to be perfect, the measured values of $\varepsilon$ indicate that our extraction system performs already quite well. The ratio $W / \varepsilon=\left(\tau-\tau_{\mathrm{e}}\right) / \tau$ according to Table 1 ranged between $49 \%$ and $73 \%$ for the various temperatures. Hence, in the present setup with unknown efficiency of the UCN detector we find that for $T=1.26 \mathrm{~K}$ at least $18 \%$ of the UCN produced in the fomblin-coated converter vessel reached the detector. Neglecting systematic uncertainties due to spectral shaping effects, for $T=0.82 \mathrm{~K}$ this number might be in the order of $W=27 \%$ for the given extraction and detection system.

It is an interesting question which saturated UCN density and which total number of trapped UCN might be achieved in near future. We first note that, for practical reasons, in the present experiments the saturated UCN density (normalised to the production volume $V_{\mathrm{p}}$ ) and the total number of $\mathrm{UCN}$ extracted from the vessel after accumulation did not yet exceed $40 \mathrm{UCN}$ per $\mathrm{cm}^{3}$, respectively, $2 \times 10^{4}$. To avoid activation of the stainless steel vessel only a quarter of the total volume was irradiated with the cold beam. Also significant divergence losses due to beam collimation were accepted, due to spatial restrictions of the experimental zone. In addition, for simplicity of these first tests, we did not yet employ a wall material with low neutron absorption and a high neutron optical potential of $250 \mathrm{neV}$ or beyond, such as Be, $\mathrm{BeO}$ or diamond-like carbon $[37,38]$. Using these materials will lead to a gain in UCN density both due to the larger energy of the storable neutrons and due to a larger storage time constant. The total number of UCN can be further increased using a much longer converter vessel. Since neutrons with wavelength $0.89 \mathrm{~nm}$ have a cross section of only $27 \times 10^{-27} \mathrm{~cm}^{2}$ in superfluid ${ }^{4} \mathrm{He}$ [39], their mean free path is about $17 \mathrm{~m}$. With a properly designed ${ }^{4} \mathrm{He}$ converter and using an existing intense cold neutron beam at a high flux reactor, UCN densities in the order $10^{4}$ per $\mathrm{cm}^{3}$ with a total $\mathrm{UCN}$ number up to several $10^{8}$ seem within reach.

Acknowledgements We are very grateful to S. Paul and W. Petry for supporting this development. We also thank I. Altarev, A. Müller and W. Schott for useful discussions. We gratefully acknowledge the design work of S. Mironov and the help of M. Pfaller and his team from the central mechanical workshop of the physics faculty for careful manufacturing of many cryostat components. This work has been funded by the German BMBF (contract number 06MT250).

Open Access This article is distributed under the terms of the Creative Commons Attribution Noncommercial License which permits any noncommercial use, distribution, and reproduction in any medium, provided the original author(s) and source are credited.

\section{References}

1. R. Golub, D. Richardson, S.K. Lamoreaux, Ultra-Cold Neutrons (IOP, Bristol, 1991)
2. V.K. Ignatovich, The Physics of Ultracold Neutrons (Clarendon Press, Oxford, 1990)

3. C.A. Baker et al., Phys. Rev. Lett. 97, 131801 (2006)

4. M. Pospelov, A. Ritz, Ann. Phys. 318, 119 (2005)

5. R.E. Lopez, M.S. Turner, Phys. Rev. D 59, 103502 (1999)

6. A. Coc, N.J. Nunes, K.A. Olive et al., Phys. Rev. D 76, 023511 (2007)

7. M. Arif et al., Precision measurements with slow neutrons. J. Res. Natl. Stand. Technol. 110, 137 (2005)

8. H. Abele, Prog. Part. Nucl. Phys. 60, 1 (2008)

9. V.V. Nesvizhevsky, H.G. Börner, A.K. Petukhov, H. Abele et al., Nature 415, 297 (2002)

10. G. Ban, K. Bodek, M. Daum, R. Henneck et al., Phys. Rev. Lett. 99, 161603 (2007)

11. A.P. Serebrov, E.B. Aleksandrov, N.A. Dovator, S.P. Dmitriev et al., Phys. Lett. B 663, 181 (2008)

12. S. Baessler, V.V. Nesvizhevsky, K.V. Protasov, A.Yu. Voronin, Phys. Rev. D 75, 075006 (2007)

13. O. Zimmer, Phys. Lett. B 685, 38 (2010)

14. A.P. Serebrov, O. Zimmer, P. Geltenbort, A.K. Fomin et al., JETP Lett. 91, 8 (2010)

15. A. Steyerl, H. Nagel, F.-X. Schreiber et al., Phys. Lett. A 116, 347 (1986)

16. U. Trinks, F.J. Hartmann, S. Paul, W. Schott, Nucl. Instrum. Meth. A 440, 666 (2000)

17. A. Fomin et al., PSI Report TM-00-14-01 (2000). See also http:// ucn.web.psi.ch/

18. A. Saunders, J.M. Anaya, T.J. Bowles et al., Phys. Lett. B 593, 55 (2004)

19. Y.N. Pokotilovski, Nucl. Instrum. Meth. A 356, 412 (1995)

20. Y. Masuda, T. Kitagaki, K. Hatanaka et al., Phys. Rev. Lett. 89, 284801-1 (2002)

21. C.A. Baker, S.N. Balashov, J. Butterworth, P. Geltenbort et al., Phys. Lett. A 308, 67 (2003)

22. The LANSCE neutron EDM experiment, http://p25ext.lanl.gov/ edm/edm.html

23. R. Golub, J.M. Pendlebury, Phys. Lett. A 53, 133 (1975)

24. P. Schmidt-Wellenburg, K.H. Andersen, O. Zimmer, Nucl. Instrum. Meth. A 611, 259 (2009)

25. O. Zimmer, K. Baumann, M. Fertl, B. Franke, S. Mironov, C. Plonka, D. Rich, P. Schmidt-Wellenburg, H.-F. Wirth, B. van den Brandt, Phys. Rev. Lett. 99, 104801 (2007)

26. A.I. Kilvington et al., Phys. Lett. A 125, 416 (1987)

27. P. Schmidt-Wellenburg, K.H. Andersen, P. Courtois et al., Nucl. Instrum. Meth. A 611, 267 (2009)

28. H. Yoshiki, K. Sakai, T. Kawai, S. Goto'o, Cryogenics 34, 277 (1994)

29. P.R. Huffman, C.R. Brome, J.S. Butterworth, K.J. Coakley et al., Nature 403, 62 (2000)

30. P. Ageron, W. Mampe, R. Golub, J.M. Pendlebury, Phys. Lett. A 66, 469 (1978)

31. Yu.N. Pokotilovski, Sov. J. Exp. Theor. Phys. 96, 172 (2003)

32. K. Zeitelhack, C. Schanzer, A. Kastenmüller et al., Nucl. Instrum. Meth. A 560, 444 (2006)

33. F. Atchison, T. Brys, M. Daum et al., Nucl. Instrum. Meth. A 552, 513 (2005)

34. M.R. Gibbs, PhD thesis, Keele University (1996)

35. M. van der Grinten, Private communication

36. W. Mampe, P. Ageron, C. Bates, J.M. Pendlebury, A. Steyerl, Phys. Rev. Lett. 63, 593 (1989)

37. F. Atchison, B. Blau, M. Daum, P. Fierlinger et al., Phys. Lett. B 642, 24 (2006)

38. F. Atchison, B. Blau, M. Daum, P. Fierlinger et al., Phys. Rev. C 74, 055501 (2006)

39. H.S. Sommers, J.G. Dash, L. Goldstein, Phys. Rev. 97, 855 (1955) 